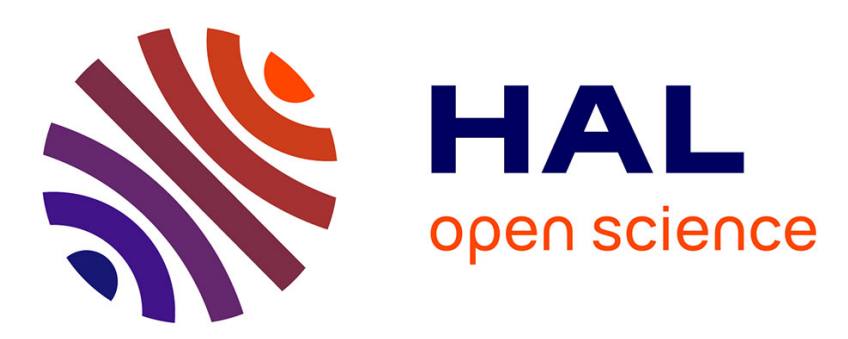

\title{
Imitation, innovation et transfert de technologie: le faciès atlantique en péninsule Ibérique à l'âge du Bronze final
}

Claire Pérez

\section{- To cite this version:}

Claire Pérez. Imitation, innovation et transfert de technologie: le faciès atlantique en péninsule Ibérique à l'âge du Bronze final. Archimède: archéologie et histoire ancienne, 2014, 1, pp.238-244. 10.47245/archimede.0001.act.06 . halshs-01586710

\section{HAL Id: halshs-01586710 \\ https://shs.hal.science/halshs-01586710}

Submitted on 13 Sep 2017

HAL is a multi-disciplinary open access archive for the deposit and dissemination of scientific research documents, whether they are published or not. The documents may come from teaching and research institutions in France or abroad, or from public or private research centers.
L'archive ouverte pluridisciplinaire $\mathbf{H A L}$, est destinée au dépôt et à la diffusion de documents scientifiques de niveau recherche, publiés ou non, émanant des établissements d'enseignement et de recherche français ou étrangers, des laboratoires publics ou privés. 


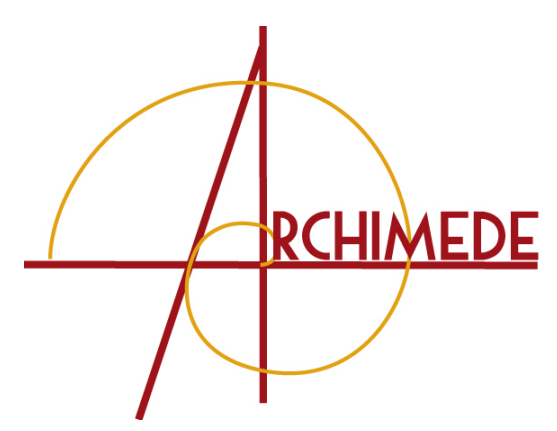

\title{
IMITATION, INNOVATION ET TRANSFERT DE TECHNOLOGIE : LE FACIĖS ATLANTIQUE EN PÉNINSULE IBÉRIQUE À L’ÂGE DU BRONZE FINAL
}

\author{
Claire PEREZ
}

Doctorante en archéologie, travaillant sous la direction de Michel Pernot et Alexis Gorgues ; UMR 5607 - Ausonius, (CNRS/ Université de Bordeaux Michel de Montaigne)

Il existe un faciès atlantique en péninsule Ibérique défini principalement par un ensemble d'objets en alliages cuivreux (armes, outils, éléments de parure,...), des pratiques communes (dépôt) et un cadre géographique précis : les régions littorales et pré-littorales de l'océan Atlantique. En outre, les découvertes récentes montrent non seulement que la façade méditerranéenne, mais, plus généralement, l'ensemble de la Péninsule, possède des objets de mêmes types et des pratiques similaires. Il s'agit alors de s'interroger sur cette répartition et sur la place de ces artefacts dans les sociétés de l'époque. Trois interrogations orientent les recherches :

- S'agit-il du même faciès, dont les limites géographiques seraient plus vastes ? L'ensemble de la production et des pratiques serait alors caractérisé par une grande similarité

- S'agit-il d'une imitation du faciès atlantique par les populations d'autres sphères culturelles ? Il existerait donc une reconnaissance de l'objet « atlantique », qu'on cherche à reproduire ou à obtenir.

- Enfin, s'agit-il d'un transfert de technologie ? Dans ce cas, ce qui intéresserait les populations n'est pas l'objet, mais plutôt l'amélioration de celui-ci : quelques éléments technologiques familiers aux hommes de l'ouest seraient adaptés sur des produits de l'est.

Une partie du corpus étudié dans le cadre de la thèse de doctorat apporte quelques arguments au débat. La méthode employée permet de valoriser et de hiérarchiser chacun des éléments descriptifs ayant servi jusque-là à établir les anciennes typologies. Ces éléments devront être comparés les uns aux autres, en respectant le paramètre de variabilité - trop souvent oublié - lié aux impondérables de l'artisanat. Nous verrons alors que la chronologie n'explique pas tout et que le facteur technologique jouait un rôle majeur pour les populations

Mots-CLÉs

Âge du Bronze final, péninsule Ibérique, faciès atlantique, mobilier métallique, transferts de technologies, cultures et techniques.
There is an archaeological facies of Atlantic regions in the west lands of Europe, mainly defined by a set of objects in copper alloys (weapons, tools, items of ornament,...), by common practices (deposit) and by a definite geographical area : the coastal and pre-coastal zones of the Atlantic Ocean. It should be noted that burial practices, domestic architecture or potteries are of different natures, which make it impossible to call it a homogeneous culture.

In the Iberian Peninsula, recent findings show that this facies is wider : not only the Mediterranean seaboard, but, more generally, the whole peninsula yield similar material and practices. Since a few years, investigators wonder about the existence of this complex and try to identify its features. It seems then important to examine the distribution and the role of these artifacts in the societies of that time. Three questions guide the research :

- Is it the same facies, whose geographical boundaries would be wider? The entire production and practices would be then characterized by a high similarity.

- Is it an imitation of the Atlantic facies, made by people from other cultural spheres? There was therefore a recognition of the 'Atlantic' object, that one sought to replicate or to obtain.

- Finally, is it a transfer of technology ? In that case, people had no interest in the object itself, but rather in its improvement : some technological elements familiar to the Western men were adapted with products coming from the East.

A part of the corpus studied in the doctoral dissertation provides some arguments in the debate. The method used allows a valuation and a ranking of each descriptive elements which served previously to establish past typologies. These elements should be compared to each other, in accordance with the variability parameter too often forgotten -, linked to the imponderables of handicraft. We will thus see that the chronology does not explain everything and Iberian Peninsula, archaeothat the technological factor played logical facies of Atlantic a major role for populations of the regions, Late Bronze Age in the Iberian technology transfers, Peninsula. 


\section{INTRODUCTION}

Plusieurs territoires européens proches de l'océan Atlantique semblent renouer contact à l'âge du Bronze final, après une période d'éloignement progressif dès la fin de l'âge du Bronze ancien. Cet espace créé par la circulation des objets, des hommes et des idées est appelé depuis le milieu des années 1950 « Complexe atlantique », « Faciès atlantique » ou encore « Age du Bronze final atlantique »[1]. Ce travail est extrait d'une thèse de doctorat en cours, portant sur la circulation des objets de types atlantiques entre les domaines atlantique et méditerranéen de la péninsule Ibérique. Seront présentés ici les différents constats sur lesquels reposent les hypothèses de ce doctorat et la réflexion méthodologique menée dans ce cadre ces trois dernières années.

Pour saisir tous les enjeux de la méthodologie proposée et des nouvelles hypothèses développées il faut revenir au contexte de formation du complexe atlantique. Sur quelles bases scientifiques repose son identification ? Quelles en sont les bornes géographiques et chronologiques ?
La péninsule Ibérique joue un véritable rôle dans la constitution de ce faciès, mais pas nécessairement celui qu'on attend, d'où un réajustement essentiel de la méthodologie de travail et des hypothèses.

\section{L'âge du Bronze final atlantique}

Les communautés du complexe atlantique présentent plusieurs points de convergence : elles partagent un espace maritime précis, un domaine géographique, physique et climatique semblable et des éléments de culture matérielle identiques. En outre, l'examen de ces témoins archéologiques indique que ces communautés possèdent des pratiques communes. La tradition du festin est partagée par la plupart des populations, de I'Irlande à la péninsule Ibérique, comme en témoigne la répartition des broches à rôtir, des crochets à viande et des chaudrons rivetés (Fig. 1). Les dépôts d'objets métalliques en milieu terrestre sec ou humide sont très courants sur I'ensemble du territoire durant tout l'âge du Bronze final. Cependant, ce même examen de la culture matérielle indique également de grandes variétés sociales,

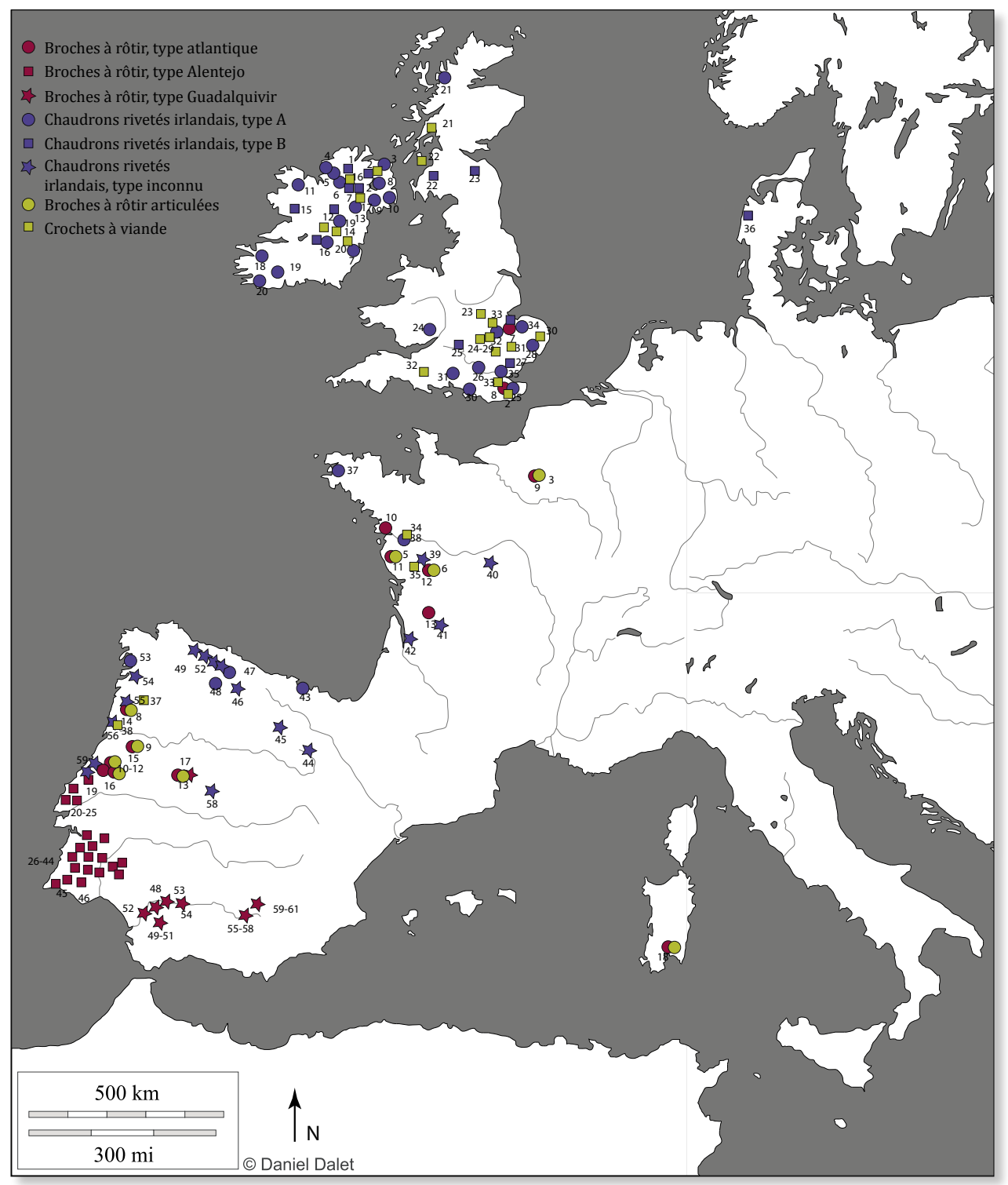

[1] MaCWhite 1951; Coffyn 1985 ; Brun 1991 ; Milcent 2012
Figure 1 :

répartition des éléments de banquet en Europe atlantique, à l'âge du Bronze final. D'après Coffyn 1985, cartes 21,22 et 28. 


\begin{tabular}{|c|c|c|}
\hline Période chronologique & Alliage & Types d'objets \\
\hline $\begin{array}{l}\text { Bronze I } \\
1250-1150 \text { a.C. }\end{array}$ & \multirow{3}{*}{$\begin{array}{l}\text { Alliage binaire } \\
\text { cuivre / étain } \\
\text { (entre } 5 \text { et } 10 \% \text { ) }\end{array}$} & Epée Ballintober, hache à talons et un anneau, pointes de lance \\
\hline $\begin{array}{l}\text { Bronze II } \\
1100-900 \text { a.C. }\end{array}$ & & $\begin{array}{c}\text { Epées pistilliformes, faucilles à douille, poignards Porto de Mós, } \\
\text { pointes de lance, haches à talon et deux anneaux, orfèvrerie } \\
\text { Berzocana, casques }\end{array}$ \\
\hline $\begin{array}{l}\text { Bronze III } \\
900-850 \text { a.C. }\end{array}$ & & $\begin{array}{l}\text { Dépôt de la Ría de Huelva, haches à douille, chaudrons rivetés, } \\
\text { crochets à viande, broches à rôtir }\end{array}$ \\
\hline $\begin{array}{l}\text { Bronze IV } \\
850-750 / 700 \text { a.c. }\end{array}$ & \multirow{2}{*}{$\begin{array}{l}\text { Alliage ternaire } \\
\text { cuivre / étain } \\
(\sim 7.5 \%) / \text { plomb } \\
\text { (entre } 50 \text { et } 75 \%)\end{array}$} & Epées de type Vénat, introduction du fer en Andalousie \\
\hline $\begin{array}{l}\text { Bronze V } \\
700 \text { a.C. }\end{array}$ & & $\begin{array}{c}\text { Haches à douille et deux anneaux, poignards à antennes et } \\
\text { épées en fer à antennes }\end{array}$ \\
\hline
\end{tabular}

Figure 2 : les types d'objets par périodes chronologiques. D'après Almagro Gorbea, 1977.

culturelles, techniques et économiques au sein de ces communautés. En raison des frontières fluctuantes, d'une culture matérielle hétérogène et des problèmes de chronologie, les chercheurs sont amenés à réfléchir à la définition de ce faciès [2].

\section{La péninsule Ibérique : province atlantique ?}

Lorsqu'en 1946 Martínez Santa Olalla identifie un âge du Bronze atlantique, il tente en réalité de souligner une rupture qui se serait opérée au début du $\mathrm{XII}^{\mathrm{e}} \mathrm{s}$. entre les origines méditerranéennes et orientales de la période précédente (1800-1200 av. J.-C.) et la véritable souche raciale du peuple espagnol [3]. L'identification d'un âge atlantique est donc fondée sur le modèle diffusionniste : le centre de rayonnement de la Péninsule est situé sur la façade atlantique qui reçoit les influences culturelles et raciales de l'Europe centrale - autrement dit des Celtes - et non plus de la Méditerranée, comme c'était le cas depuis le Néolithique [4]. Au début des années 1950, MacWhite reprend à son compte l'expression « d'âge du Bronze atlantique ». Il critique la vision diffusionniste - et raciste - de Santa Olalla mais ne réfute pas l'idée d'un âge atlantique. Au contraire, il propose une approche historico-culturelle en identifiant plusieurs groupes qui partagent des modèles précis (épées, haches, parure, vaisselle...) dont la diffusion au sein du complexe relève de contacts entre les peuples par voie maritime et non de la colonisation par d'autres cultures [5]. Il remarque en outre que les relations entre le domaine atlantique et la Péninsule sont antérieures à I'âge du Bronze final. Il met en relation les cultures mégalithiques de I'Andalousie et de I'Irlande, et souligne la correspondance entre certains artefacts (haches, plaquettes, idoles) qui présentent des formes et des décorations similaires aux modèles irlandais [6]. Une vingtaine d'années plus tard, Almagro Gorbea affine le découpage géographique en prônant une approche évolutionniste. Il existe pour cet auteur cinq aires distinctes occupées de 1250 à 700 av. J.-C., c'est-à-dire de l'âge du Bronze final I à l'âge du Bronze final IV [7]. À chaque période est recensé un type d'objet particulier composé d'un alliage cuivreux qui lui est propre (Fig. 2). Les publications des années 1980 de Coffyn et Ruiz Gálvez [8] ont permis de valoriser l'ancienneté de ces relations en démontrant que les liens entre la Péninsule et le reste du complexe remontaient au moins à l'âge du Bronze ancien. L'inventaire dressé par Coffyn permet de visualiser la répartition des vestiges en péninsule Ibérique : le quart ouest du territoire est identifié comme province atlantique (Fig. 3) [9].

En réalité, un constat fondé sur la répartition des vestiges de types atlantiques datés de l'âge du Bronze final
[2] Cette problématique relève de la définition des termes culture, communautés, groupes, complexe, faciès... Dans ce court exercice il semble hors-propos d'insister sur ce débat. Pour une première approche de la discussion on conseillera de se référer aux actes du colloque de Lisbonne : Existe uma idade do Bronze Atlântico ? (Susana Oliveira Jorge (éd.) 1998).

[3] Martínez Santa-Olalla 1946: La thèse raciale, et raciste, est la thèse principale défendue dans l'ensemble de son ouvrage.

[4] Martínez Santa Olalla était l'étudiant de Bosch Gimpera. Il était très proche des théories indo-européennes de Gustaf Kossina et de la pensée nazie. Il a effectué plusieurs cycles de conférences en Allemagne, sur invitation d'Heinrich Himmler. L'essentiel de sa pensée tend à prouver l'origine indo-européenne de l'Espagne, tout en refusant l'existence du peuple ibère (Mederos Martín 2003, p. 13-56).
[5] MACWhite 1951. Même si les témoins archéologiques manquent - principalement à son époque - il s'appuie sur les quelques exemplaires de barques retrouvées en Europe centrale et en Irlande pour démontrer l'importance de la navigation dès le Néolithique.

[6] MAcWhite 1951, p. 20-28, fig. 4.

[7] Almagro-Gorbea 1977 ; 1986. L'auteur propose pour la fin de l'âge du Bronze de la péninsule Ibérique, une chronologie en cinq phases. Bronze final I : 1250-1150 av. J.-C.; Bronze final II : 1150900 av. J.-C. ; Bronze final III : 900-850 av. J.-C. ; Bronze final IV : 850-750/700 av. J.-C. ; Bronze final V : 700 av. J.-C.

[8] Coffyn 1985 ; Ruiz-Gálvez Priego 1984.

[9] COFFYN 1985. 


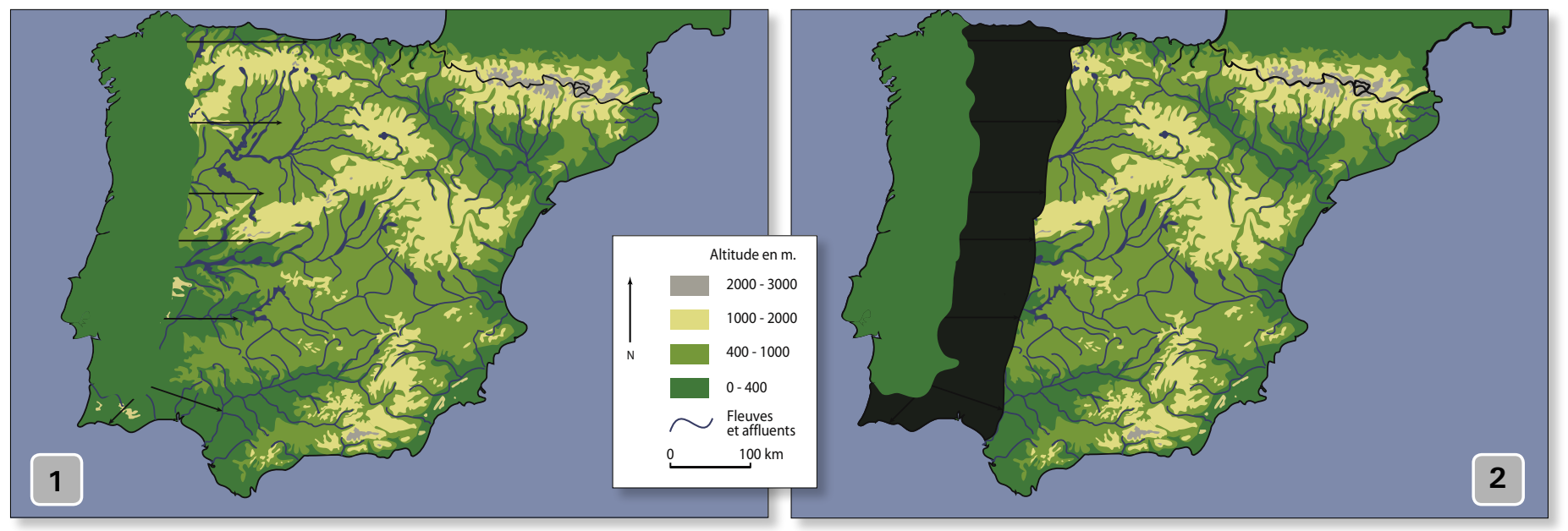

Figure 3 : l'évolution géographique du faciès atlantique, en péninsule lbérique à l'âge du Bronze final.

1 : frontières définies par Santa Olalla puis MacWhite au milieu du Xxe siècle. 2 : La répartition des vestiges réputés de types atlantiques permet à Coffyn d'élargir les frontières du faciès au quart ouest de la péninsule lbérique. Cartes de l'auteure.

nous permet de remettre en cause les limites du faciès généralement admises, cantonnées au quart ouest de la Péninsule.

\section{LE CONSTAT}

Coffyn propose des cartes de répartition des vestiges de l'âge du Bronze final atlantique qui montrent I'extension du faciès vers les zones littorales de la Méditerranée [10] (Fig. 4). Ces objets sont considérés comme des exceptions ce qui tend à démontrer que les populations des territoires de l'Est imitaient ou importaient des objets normalement fabriqués ou diffusés dans les territoires atlantiques habituels [11].

En réalité, la présence de ces objets n'est pas anecdotique même si la concentration des vestiges est moins importante vers la Méditerranée que dans les territoires de l'Ouest. Cela ne s'explique pas uniquement par la position périphérique des zones méditerranéennes par

Figure 4 : répartition des objets réputés de types atlantiques, en péninsule lbérique, à l'âge du Bronze final. Carte de l'auteure.

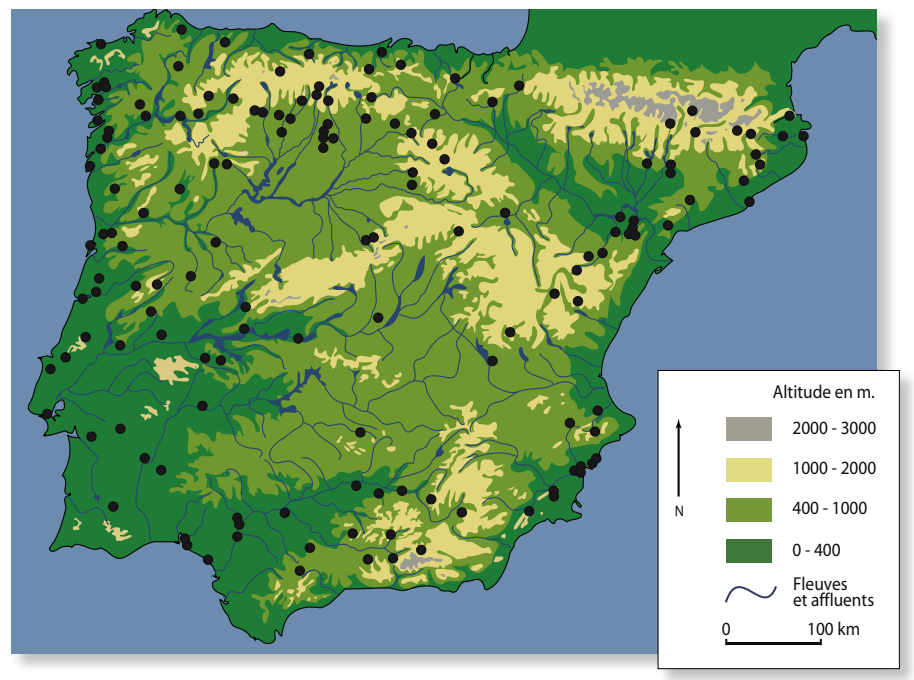

rapport aux territoires habituels. Pour comprendre cette répartition il faut également prendre en compte les différents contextes de découverte, les cadres chronologiques de chaque vestige et le développement de la recherche scientifique dans les régions et départements actuels.

Deux catégories d'objets se distinguent : les différents types de haches et les épées. Quelques rares pointes de lances sont recensées, très peu de poignards, et des éléments de parure souvent peu caractéristiques d'un type ou d'une période. Cette prédominance des haches et des épées est également visible dans le quart ouest de la péninsule Ibérique, considéré comme le berceau du faciès. La proportionnalité entre les différents types de vestiges est la même à l'ouest et à l'est, même si le nombre de découvertes est manifestement moindre vers la Méditerranée (Fig. 4).

La répartition des vestiges s'organise autour de trois gros foyers, différents du quart ouest de la Péninsule. Le premier longe la côte entre les Pyrénées et l'embouchure de l'Ėbre et s'étend le long du fleuve en direction de la Rioja. Le deuxième foyer est situé en Andalousie, le long du Guadalquivir et de ses affluents. Le troisième foyer est très proche, il se développe le long de la côte méditerranéenne, entre Almería et le sud de Valencia. On retrouve quelques vestiges également le long du Tage et des ses affluents, au pied du Système Central.

Constater l'éclatement des frontières et la présence de vestiges à l'opposé des zones habituellement identifiées comme atlantiques n'est pas suffisant. Il faut maintenant caractériser les relations qui existent entre les vestiges de l'ouest et du reste de la péninsule Ibérique.

[10] COFFYn 1985.

[11] Harrison 1974, p. 229 ; Alonso Fernández \& Jiménez ECHEVARRÍA 2009, p. 21-22. 


\section{POUR UNE AUTRE RÉFLEXION MÉTHODOLOGIQUE}

\section{QUELS SONT LES LIENS ENTRE LES OBJETS DE TYPES ATLANTIQUES DE LA PÉNINSULE IBÉRIQUE?}

Les découvertes récentes montrent que la façade méditerranéenne et, plus généralement, l'ensemble de la Péninsule, possèdent également des objets de même type et des pratiques similaires. Il s'agit alors de s'interroger sur cette répartition et sur la place de ces artefacts dans les sociétés de cette époque. Trois interrogations orientent les recherches :

- Ces objets sont-ils des productions atlantiques typiques à l'intérieur d'un complexe plus vaste dont on aurait mal saisi les frontières au premier abord ? L'ensemble de la production et des pratiques serait alors caractérisé par une grande similarité. La péninsule Ibérique dans son intégralité pourrait être identifiée comme une province atlantique.

- S'agit-il d'une imitation des types atlantiques par des populations d'autres sphères culturelles ? Il existerait donc une reconnaissance de l'objet « atlantique », qu'on cherche à reproduire ou à obtenir. Ce schéma expliquerait la présence de moules dans les territoires «non atlantiques », c'est-à-dire en dehors du quart ouest de la Péninsule, et permettrait d'accepter également l'importation de certains artefacts.

- Enfin, s'agit-il d'un transfert de technologie ? Dans ce cas, ce qui intéresse les populations n'est pas l'objet, mais I'amélioration de celui-ci : quelques éléments technologiques familiers aux hommes de l'ouest sont adaptés sur des produits de l'est afin d'obtenir une « efficacité technique » supérieure aux modèles indigènes. Ce schéma permet de développer de nouveaux types, des sortes d'artefacts hybrides pour lesquels on retrouve des éléments technologiques du faciès atlantique et des éléments souvent moins fonctionnels, qui sont liés au fonctionnement interne des communautés [12].

Des éléments de réponse peuvent être déduits des recherches précédentes, mais elles présentent certaines limites qu'il faut caractériser et tenter de dépasser.

\section{DES MÉTHODES QUI ONT FAIT LEUR PREUVE}

Actuellement l'étude de l'âge du Bronze final atlantique repose sur des chrono-typologies qui mettent en évidence de possibles groupes culturels et chronologiques. Ces chrono-typologies sont fondées sur des critères purement descriptifs : la forme de la garde des épées, la section de l'embouchure des haches à douille, la présence d'un ou plusieurs anneau( $x$ ) sur les haches, la longueur de la flamme des pointes de lance, le système de fixation des poignées d'épées, etc. Il ne s'agit pas de mettre de côté ces critères qui ont prouvé qu'ils étaient en partie efficaces : par cette méthode, les épées à langue de carpe de type Huelva, caractéristiques de la fin de l'âge du Bronze en péninsule Ibérique, ont pu être distinguées des épées de type Nantes, qui, elles, sont caractéristiques du faciès en langue de carpe de la France et de la Grande-Bretagne, daté de I'âge du Bronze final III [13]. Un calage chronologique précis a été établi pour l'âge du Bronze final atlantique de la Gaule en se fondant sur la description des objets et en les comparant à leurs homologues ibériques et britanniques [14].

\section{POUR ALLER PLUS LOIN}

La limite principale de cette méthode descriptive est la multiplication de types différenciés dans certains cas par des éléments peu pertinents. Des variations dans un groupe d'objets qui se ressemblent ne s'expliquent pas nécessairement par la volonté de produire un objet différent. Ce procédé présente également le désavantage de retirer l'objet de son contexte de fabrication et d'usage, notamment en excluant le contexte de découverte de I'analyse. En résumé, cette méthode étudie l'objet trouvé, le vestige archéologique et non l'objet utilisé. Les problématiques soulevées dans ce travail autorisent une réflexion méthodologique parallèle, qui repose sur ce système de description en intégrant d'autres paramètres qui prennent en compte les éléments liés au contexte général de vie des objets [15].

Concrètement, les informations contenues dans la base de données ont été rassemblées dans trois tableaux (un pour les haches, un pour les épées et un pour les pointes de lance). Ces tableaux ont été élaborés en respectant deux principes :

- Les sous-catégories d'objets (différents emmanchements des haches, différentes lames des épées) introduisent un biais méthodologique parce qu'elles empêchent

[12] En d'autres termes il s'agit d'éléments ou de comportements qui apparaissent illogiques aux yeux des chercheurs actuels car ils ne répondent pas aux modèles sociaux que nous connaissons. Les mécanismes de construction des communautés de l'âge du Bronze sont plus abstraits pour les chercheurs actuels car moins visibles, mais pourtant, ils ont un impact direct sur les vestiges matériels. Il peut s'agir par exemple d'éléments décoratifs ou de la conservation d'anciens outils considérés comme traditionnels alors même que d'autres seraient plus efficaces.

[13] BrandHerm \& MosKal-DEL-Hoyo 2010, p. 432

[14] MiLCENT 2012 et particulièrement les pages 166-169.

[15] Le contexte général de vie correspond à toutes les étapes que l'objet va connaître : de sa fabrication à son abandon et sa redécouverte pas les archéologues. 
de développer une réflexion sur la fonction, sur l'efficacité technique ou sur la symbolique des objets. Ces souscatégories sont donc, dans un premier temps, abolies.

- Le deuxième grand principe permet d'intégrer ces réalités liées à la fabrication et à l'usage des objets à I'intérieur des tableaux. Les critères de description qui sont choisis doivent permettre de redonner une place majeure à l'objet utilisé et non à l'objet trouvé. Ces réalités interviennent à chaque étape de la vie d'un objet, de sa conception à son dépôt.

\section{DE LA CONCEPTION AU DÉPÔT D'UN OBJET : DIFFÉRENTES RÉALITÉS QUI INFLUENT SUR LA FORME DU VESTIGE}

Différencier le vestige de l'objet utilisé apparaît comme le point central de la réflexion méthodologique proposée ici. L'artefact va subir plusieurs étapes depuis sa conception jusqu'à sa découverte qui vont influer sur la forme du vestige. Ces étapes correspondent à différentes réalités - techniques, artisanales, sociales, économiques ou taphonomiques - qu'il faut définir et expliquer pour comprendre l'objet trouvé.

\section{La réalité technologique}

Un outil ou une arme doivent répondre à certains critères censés optimiser leur utilisation, en s'adaptant au mieux aux activités pour lesquelles ils sont conçus (dans le cas où ce n'est pas la forme de l'objet qui détermine son usage).

De même qu'on distingue la forme d'une épée destinée à frapper de taille (avec la tranche) ou d'estoc (avec la pointe), on distingue également les armes destinées au combat rapproché, des armes de jet, destinées à blesser à distance.

\section{La réalité artisanale}

La valeur artisanale, c'est-à-dire la facilité variable avec laquelle les artisans fabriquent la pièce est étroitement liée à la valeur technologique. Les artisans vont rencontrer un certain nombre de limites pour fabriquer leurs objets qui vont parfois déterminer leur usage.

- Les conditions physiques (espace de travail, matériel) et climatiques dans lesquelles ils vont réaliser l'objet.

- Les savoir-faire dont ils disposent, leur expérience, leur degré d'agilité, qui vont influer sur la qualité de l'alliage, la facture du moule, la finesse des décors et des finitions, les dimensions de l'objet etc. La différence de masse ou de longueur des objets peut s'expliquer par l'absence de standardisation ou de production à la chaîne. Même si les artisans souhaitent fabriquer plusieurs moules à la même effigie ou fondre plusieurs objets dans le même moule il semble évident que des décalages vont se créer, lors de la fonte ou des étapes de finition. Ces différences que nous observons à la loupe étaient certainement peu visibles pour les populations de l'âge du Bronze.

\section{Les réalités sociales, culturelles, idéologiques ...}

Ces valeurs sont particulièrement difficiles à appréhender. Il ne s'agit pas d'éléments purement fonctionnels, au moins au bout d'un certain temps. Ce sont des éléments qui vont renvoyer à un lignage, à une croyance ou à une tradition. Ils peuvent devenir systématiques sans pour autant créer un dysfonctionnement dans le cas où ils disparaitraient. Certaines haches à douille illustrent très bien cette idée : leur extrémité proximale est décorée par des simulacres d'ailerons en relief qui rappellent les haches à ailerons - élément technologique qui finit par passer dans la tradition décorative. Certaines de ces valeurs vont être appréhendées plus facilement tandis que les autres resteront dans la partie invisible de I'histoire archéologique.

\section{La réalité économique}

La réalité économique est celle prise en compte lors d'un échange, vente, prêt, calcul de la richesse d'une personne ou d'une communauté, coût de fabrication de la pièce. Elle peut être liée à la valeur précédente en ce sens que sa valeur marchande augmentera ou diminuera en fonction de la symbolique de l'objet. Au contraire elle peut être beaucoup plus concrète et dépendre de l'alliage (couleur de l'objet), de la masse ou de la taille des artefacts.

\section{La réalité taphonomique}

Ce phénomène intervient après l'abandon ou le dépôt de l'objet. Il s'agit de tous les phénomènes, anthropiques ou naturels, qui vont affecter l'objet à l'intérieur de son contexte stratigraphique. Pour plusieurs raisons ce dernier peut varier au cours du temps : terre remuée par I'homme ou les animaux, effondrement du terrain, courants maritimes, alluvions, précipitations. Lorsque la position stratigraphique de l'objet ne change pas elle peut alors renseigner efficacement sur la fonction de I'objet. Cette réalité peut s'observer à l'œil nu sur le vestige : corrosion, rouille, fractures, disparition des matières périssables (bois, os, tissus...) ou des décors, rayures, impacts...

L'ensemble de ces réalités, confrontées les unes aux autres, doit permettre de mieux comprendre la structure de la société de l'âge du Bronze final. Elle doit faciliter l'identification d'éventuels groupes qu'il s'agisse de groupes culturels, technologiques ou économiques. 


\section{ÉLÉMENTS DE CONCLUSION}

Nous avons vu que la démarche évolutionniste et l'approche typo-chronologique ne permettent pas de prendre pleinement conscience des contraintes qui encadrent la production d'un objet métallique. Ainsi, les variations qui existent d'un objet à l'autre peuvent être le reflet de techniques artisanales différentes ou de besoins culturels et technologiques variés. Ces variations ne sont pas systématiquement le résultat d'une évolution naturelle fondée sur la recherche de la performance et de l'amélioration. Ainsi, les premières conclusions qui se dessinent permettent d'ouvrir une réflexion sur l'utilisation des épées, des haches et des pointes de lance au cours de l'âge du Bronze final. Dans le premier cas on notera une recherche d'individualisation marquée, nettement appuyée par les contextes de découvertes qui donnent de l'importance à l'objet, en l'isolant du reste du matériel ou par exemple en l'exposant dans des lieux stratégiques : grottes, rochers, contenants divers, etc. La fabrication d'une hache, et a fortiori son utilisation démontrent au contraire une grande variété de types et de formes, en partie liée à la chronologie mais principalement issue des traditions techniques, artisanales et culturelle des différents acteurs. En outre, ces différents choix apparaissent être également déterminés par la fonction première de l'objet : arme ou outil. Concernant les pointes de lance, la réflexion évolue au fur et à mesure qu'elle s'éloigne des préjugés concernant I'utilisation de l'artefact. À terme, nous espérons proposer des pistes sérieuses permettant d'aborder la place et la fonction de cet objet au sein du répertoire métallique de la fin de l'âge du Bronze : s'agit-il d'une arme de jet, fonctionnelle, solide et performante?

Enfin, la nouvelle démarche permet de prendre la mesure de l'intégration de la péninsule Ibérique dans le monde atlantique. Le développement de types d'objets particuliers est le premier argument plaidant pour un rôle actif de la Péninsule face aux autres régions européennes. Cette piste de réflexion doit être menée en perspective des études typo-chronologiques pour tenter d'identifier les réseaux d'échanges, d'imitations et d'innovations dans lesquels s'inscrit la péninsule Ibérique de la fin de l'âge du Bronze.

Les conclusions détaillées de ce travail ont été présentés en soutenance le vendredi 20 décembre 2013 et feront l'objet de publications ultérieures.

Almagro-Gorbea, M., 1977, El Bronce final y el período orientalizante en Extremadura, Madrid.

Almagro-Gorbea, M., 1986, Bronce final y Edad del Hierro. La formación de las etnias y culturas prerromanas, in F. Jordá Cerdá (éd.), Historia de España. Prehistoria-1, Madrid, p. 341-532.

Alonso Fernández, C., Jiménez Echevarría, J., 2009, «El depósito de armas del bronce final de "Los Cascajos", Grañón (La Rioja) », Gladius 29, p. 7-38.

Brandherm, D. \& Moskal-del-Hoyo, M., 2010, «Las espadas en lengua de carpa - aspectos morfológicos, matalúrgicos y culturales », Trabajos de Prehístoria 67, 2, p. 431-456.

Brun, P., 1991, « Le Bronze atlantique et ses subdivisions culturelles : essai de définition », in C. Chevillot et A. Coffyn (éd.), L'âge du Bronze atlantique: ses faciès, de l'Écosse à l'Andalousie et leurs relations avec le Bronze continental et la Méditerranée, Actes du $1^{\text {er }}$ colloque du parc archéologique de Beynac, Beynac, p.11-24.

Coffyn, A., 1985, Le Bronze final atlantique dans la Péninsule ibérique, Université de Bordeaux III, Centre Pierre Paris, 441 p. Harrison, J.R., 1974, « Nota acerca de algunas espadas del bronce final en la Península Ibérica », Ampurias 36, p. 225-234.

MacWhite, E., 1951, Estudios sobre las relaciones atlánticas de la Península Hispánica en la Edad del Bronce, Madrid, Seminario de Historia primitiva del Hombre (Disertaciones Matritenses, II), $151 \mathrm{p}$.

Martínez Santa-Olalla, J., 1946, Esquema paletnológico de la península hispánica, Diana, 1946

Mederos Martín, A., 2003, «Julio Martínez Santa-Olalla y la interpretación aria de la Prehistoria de España (1939-1945) 》, Boletín del Seminario de Estudios de Arte y Arqueología 69, p. 13-56.

Milcent, P.-Y., 2012, Le temps des élites en Gaule atlantique: chronogie (sic) des mobiliers et rythmes de constitution des dépôts métalliques dans le contexte européen, $x_{I I I^{e}}$-VII ${ }^{e}$ av. J.-C., Rennes (Archéologie et culture), 253 p.

Ruiz-Gálvez Priego, M., 1984, La Península Ibérica y sus relaciones con el círculo cultural atlántico, Universidad Complutense, Madrid. 